\section{Promoção da saúde: perspectivas avaliativas para a saúde bucal na atenção primária em saúde}

\author{
Health promotion: perspectives for evaluation of \\ oral health in primary healthcare
}

\author{
1 Pontificia Universidade \\ Católica do Paraná, Curitiba \\ Brasil. \\ Correspondência \\ S. Z. Kusma \\ Pontifícia Universidade \\ Católica do Paraná. \\ Rua Antonio Freitas Barbosa \\ 44, Curitiba, $P R$ \\ 81110-110, Brasil. \\ solkusma@yahoo.com.br
}

\section{Abstract}

The evaluation of health promotion activities is a methodological and strategic challenge for establishing evidence to support health management processes. The use of adequate evaluation methods based on participatory analysis of local processes and contexts is essential to the success of interventions and policy formulation and implementation. Brazil's Policy for Oral Health Promotion and Surveillance explicitly states the need to improve evaluation strategies for oral health promotion activities conducted in the context of primary care, allowing to evaluate not only their results and impact, but also the political and social process for achieving the objectives. This article proposes to systematize the literature on the evaluation of effectiveness in health promotion strategies, define a theoretical model, and propose a matrix of descriptors, exploring the basic references for health promotion and practices with the potential to reduce situations of vulnerability in population groups, combat inequalities, and incorporate community participation in health management.

Health Promotion; Primary Health Care; Oral Health
Solena Ziemer Kusma 1

Simone Tetu Moysés 1

Samuel Jorge Moysés 1

\section{Introdução}

A área da saúde bucal no Brasil, como parte integrante da atenção primária à saúde e das ações de vigilância em saúde, depara-se com desafios importantes para responder com efetividade às situações e problemáticas que exigem enfrentamento cotidiano. Tais desafios, de natureza teórico-metodológica, prática e avaliativa, manifestam-se tanto na necessidade de demonstrar resolutividade na assistência clínica (tão necessária à maioria dos brasileiros), como na formulação de políticas intersetoriais sustentáveis, de natureza mais abrangente e que possam impactar positivamente os principais indicadores epidemiológicos de saúde bucal 1 .

Nos últimos anos, observa-se um esforço para promover uma maior integração da saúde bucal aos serviços de saúde em geral, possibilitando a sinergia de saberes e práticas que apontem para a promoção e vigilância em saúde, atuação sobre determinantes sociais do processo saúdedoença, prevenção de riscos e doenças, e a consequente incorporação de práticas baseadas em evidências de efetividade 2 .

Entretanto, boa parte das práticas anunciadas como de "promoção da saúde", sobretudo no nível da atenção primária, ainda são limitadas a estratégias baseadas em modelos tradicionais de intervenções educativo-preventivas centradas em higiene bucal supervisionada, palestras, aplicações de fluoretos, desenvolvidas priori- 
tariamente em ambientes escolares ${ }^{3}$. Algumas dessas intervenções sabidamente apresentam problemas em sua operacionalização e na força de evidência científica, caracterizando ações divergentes e inconscientes ${ }^{4}$. Revisões sistemáticas da literatura têm reafirmado a baixa força de evidências quanto ao impacto de intervenções preventivas/educativas convencionais no campo da odontologia 5,6,7,8,9. Algumas das mais sérias limitações dessas ações se devem ao fato de não produzirem melhorias sustentáveis em médio e longo prazo na saúde bucal de populações, sendo paliativas em sua natureza, ignorando amplamente os fatores estruturais que determinam uma saúde bucal deficiente. Paradoxalmente, um dos possíveis resultados dessas ações focalizadas em indivíduos é que as iniquidades, ao contrário de serem reduzidas, podem ser agravadas, já que aqueles que têm mais recursos (materiais, cognitivos, contextuais) estão mais aptos a serem beneficiados pelas intervenções executadas. Esse não é um problema exclusivo da saúde bucal, pois outras áreas da saúde têm destacado as mesmas limitações da abordagem clínica preventiva ou das práticas educativas mais individualizadas 10,11 .

Entender a promoção da saúde como estratégia para o processo de reformulação de práticas sanitárias emerge, portanto, como o ponto de partida para o enfrentamento de desafios. A promoção da saúde, além de ser uma das ações estratégicas da vigilância em saúde, é um dos eixos centrais estabelecidos pelo Sistema Único de Saúde (SUS) para a construção de uma abordagem integral do processo saúde-doença 12.

Nesta linha, é lícito advogar por uma promoção da saúde bucal que busque alcançar melhoras sustentáveis neste campo de atividade humana, reduzindo iniquidades por meio de ações direcionadas a seus principais determinantes 10,13. Isso implica no desenvolvimento de ações multidimensionais e intercomplementares, de base populacional, que potencializem fatores protetivos (positivos) de saúde. Tais ações são comprometidas com o direito à saúde, à equidade $\mathrm{e}$ à cidadania, assim como voltadas ao desenvolvimento humano e a proteção social, mantendo uma fina sintonia com as necessidades e demandas de saúde de indivíduos e populações em territórios e grupos populacionais específicos, contextualizadas de acordo com suas condições de vida.

Desenvolver ações nesta direção exige o estabelecimento de uma agenda de políticas públicas saudáveis como referência central para a ação institucionalizada em saúde bucal, fortalecendo estruturas e processos comprometidos com a promoção da saúde. Além disso, é neces- sária uma proativa busca de conhecimento para fundamentar as práticas de promoção da saúde, o que impõe o investimento consistente no desenvolvimento da força de trabalho em saúde, e a capilarização com busca de legitimação social sobre a importância das estratégias de promoção da saúde bucal 14 .

Cabe aos serviços, então, ampliar o entendimento da promoção da saúde no contexto da atenção primária, estabelecendo-o como referência para todo o processo de trabalho, expandindo o conceito de saúde para além da assistência a pessoas doentes, promovendo a qualidade de vida por meio de intervenções sobre os fatores que colocam a população em risco 15 .

Semelhantes ações incluem os indivíduos, suas famílias, e os territórios geopopulacionais onde vivem e trabalham, tendo em conta um amplo arco de possibilidades individuais e comunitárias que passam por geração de trabalho e renda dignos, provimento de habitação e alimentação suficientes e saudáveis, saneamento adequado, lazer e cultura da paz e da não violência, dentre outros. Elas objetivam, ,mediante defesa da saúde, fazer que as condições de vida saudável sejam cada vez mais possíveis, favorecendo escolhas pessoais em prol da saúde. As cidades, os locais de trabalho, as escolas, os territórios operativos das Equipes de Saúde da Família são exemplos de ambientes onde tais ações têm sido implementadas, visando o fortalecimento do protagonismo do nível local, incentivando a equidade, a colaboração intersetorial e a participação popular 16,17,18.

Embora se reconheçam avanços na incorporação de novos conhecimentos e nas práticas de promoção da saúde bucal na última década, na esfera pública, ainda assim um conjunto desafiador de problemas envolvendo o perfil epidemiológico das doenças bucais e a resolutividade da atenção permanece vivo no debate político, científico e de gestão de serviços.

A Organização Mundial da Saúde (OMS), em seu relatório Saúde Bucal Mundial - 2003, enfatizou que, apesar de grandes melhorias no estado de saúde bucal de populações em período recente, problemas graves ainda persistem ${ }^{19}$. O relatório aponta como desafios principais no futuro a tradução do conhecimento já existente e experiências efetivas de prevenção de doenças e promoção da saúde em programas de ação estratégica apoiados nas evidências sobre melhores práticas, contextualizadas nos territórios de atuação.

A atual Política Nacional de Promoção de Saúde no Brasil aponta como responsabilidade das três esferas de governo o estabelecimento de instrumentos e indicadores para o acompanha- 
mento e avaliação do impacto/implementação dos objetivos definidos na referida política. Os instrumentos deveriam estar centrados nos princípios e valores da promoção da saúde, incluindo a integralidade, equidade, responsabilidade sanitária, mobilização e participação social, intersetorialidade, informação, educação e comunicação, além da sustentabilidade. A política ainda aponta como uma de suas diretrizes o incentivo à pesquisa em promoção da saúde e a divulgação de iniciativas efetivas para profissionais de saúde, gestores e usuários do SUS, considerando metodologias participativas e o saber popular 20.

Nesta mesma perspectiva e como decorrência da implementação da Política Nacional de Saúde Bucal centrada na promoção e na vigilância em saúde bucal, demanda-se o aprimoramento e investimento em estratégias avaliativas que suportem a tomada de decisão na gestão de serviços de saúde bucal. Avaliar práticas com o objetivo de apoiar a gestão da atenção primária em saúde implica o uso de metodologias que permitam avaliar não somente os resultados e impacto das intervenções, mas também o processo político-social para alcançar os objetivos de forma sustentável 21 .

Com base nessas premissas, este artigo objetiva sistematizar a literatura no campo da avaliação de efetividade de estratégias de promoção da saúde bucal, definir um modelo teórico e propor uma matriz de descritores, explorando a base referencial dos pilares e valores da promoção da saúde contextualizados nas práticas de atenção primária em saúde.

\section{Avaliação em promoção da saúde: um desafio metodológico e estratégico}

A avaliação de ações de promoção de saúde traz um complexo desafio metodológico 22 . Avaliação constitui um recurso técnico e político relevante para a busca de reorientação da racionalidade das práticas de saúde 23 . Pode ser considerada um julgamento sobre uma prática social ou qualquer dos seus componentes, em diferentes contextos e espaços sociais, com o objetivo de auxiliar na tomada de decisão informada, apoiando-se em métodos robustos que respondam adequadamente a formulações teórico-políticas que guiam as estratégias de intervenção na realidade. Quando a avaliação assume um caráter participativo, ela cria um ambiente virtuoso, sendo a própria circunstância avaliativa um processo de empoderamento de sujeitos 24,25,26.

A demanda por metodologias avaliativas robustas, adequadas aos objetivos, contextos e cenários específicos da atenção primária em saúde e da vigilância em saúde. vem se tornando indispensável tanto para o sucesso dessas intervenções, quanto para a análise de processos locais e contextos para a formulação e implementação de práticas. Processos avaliativos de práticas de promoção da saúde podem dar suporte à construção de melhores práticas, produzindo e sistematizando conhecimentos, respondendo às demandas por novos referenciais e propostas de intervenção indutoras de mudanças em realidades locais de exclusão e dependência 27,28,29.

Segundo De Salazar 21, quando nos referimos à efetividade, estamos avaliando se uma intervenção serve para o que foi criada, quando funciona em condições reais não controladas. Nutbeam 30 caracteriza a promoção da saúde efetiva como aquela que conduz a mudanças nos determinantes da saúde. Para avaliar a efetividade das práticas, no contexto real de seu desenvolvimento, é preciso utilizar indicadores que incluam não apenas resultados em saúde, mas apropriação, viabilidade e sustentabilidade do processo político que produz mudança, bem como a capacidade coletiva, institucional e territorial (local) para intervir. Deve ainda incluir indicadores de fatores de risco e determinantes sociais em saúde, equidade, resultados intermediários associados, equilíbrio entre relações de poder e participação social para decisões negociadas que decorrem de governança pública responsável, afetando em última instância a saúde da população. Mais ainda, para avaliar tais práticas é preciso reconhecer que o contexto sociopolítico tem efeito modificador nos resultados. Isso explica a dificuldade de fazer afirmações precisas sobre o benefício de uma prática, em termos de mudança real para as condições de vida e saúde e a atribuição dos resultados para uma intervenção específica 31,32 .

Intervenções de promoção da saúde bucal envolvem dimensões complexas, definidas pela variedade de contextos, grupos sociais e instituições, abrangendo a colaboração e participação de diferentes setores e atores no território e a utilização de múltiplas estratégias. Além disso, são intervenções em permanente mudança e com efeitos em longo prazo. Essa complexidade tem implicações diretas sobre a forma de medir seu impacto e efetividade, pela necessidade de mensurar princípios e valores e dificuldades de generalização e produção de resultados 27,31 .

Logo, a valoração de sucesso de uma estratégia de promoção da saúde bucal deve centrar-se na sua utilidade, considerando os determinantes socioambientais associados à ganhos em saúde, bem como na possibilidade de identificação de melhores práticas que expressem valores, possibilitem a construção de evidências, a compre- 
ensão do contexto e a identificação de práticas e processos organizacionais sustentáveis.

\section{Metodologias avaliativas: tendências de avaliação em promoção da saúde}

As características de estratégias de promoção da saúde têm direcionado o debate sobre quais as melhores abordagens metodológicas para o alcance dos objetivos de processos avaliativos dessas práticas. Abordagens centradas no referencial da epidemiologia clínica, focadas na representatividade da amostra, estandardização das intervenções, valoração da magnitude dos resultados e probabilidades baseadas em testes de significância estatística e controle de fatores de confusão, não obrigatoriamente possibilitam a valoração da efetividade de práticas complexas, como as de promoção da saúde bucal 31 . Por conseguinte, o processo avaliativo de tais práticas não envolve apenas a coleta, análise e interpretação de dados quantitativos para comparação de resultados, mas também a triangulação metodológica visando a ações múltiplas e complexas associadas ao desenvolvimento da avaliação 33,34,35. Há consenso sobre a necessidade de combinação de diferentes enfoques e métodos na avaliação de estratégias de promoção da saúde que permitam a análise de seu fundamento teórico como prática social, do processo de implementação, impacto e resultados em curto, médio e longo prazos. A integração de técnicas avaliativas, como a triangulação de métodos, fontes e pesquisadores; a utilização de enfoques quantitativos e qualitativos com foco em abordagens participativas e narrativas; bem como a articulação da avaliação para processos de documentação, sistematização e monitoramento, com base em sistemas de informação tradicionalmente vinculados aos sistemas de vigilância em saúde, têm caracterizado as tendências metodológicas para avaliação de efetividade de promoção da saúde 36,37 .

Mais que uma abordagem política e estratégica, a avaliação de práticas de promoção da saúde será mais efetiva quanto mais participativas forem as metodologias utilizadas, buscando envolver no processo avaliativo quem teve ou tem alguma interferência na implementação da prática avaliada e que pode influenciar seus resultados 10. Entretanto, o envolvimento de diferentes atores sociais pode gerar conflitos de interesses no processo avaliativo, uma vez que os interlocutores passam a ser alvo da observação e devem tomar parte de todas as etapas da investigação: na sua concepção, na definição do problema, na territorialização, no levantamento de dados, nas análises do material coletado e na elaboração dos resultados 32 . Consequentemente, o processo avaliativo pode fortalecer capacidades para a ação e busca de interesses comuns, tornar oportunas reflexões e aprendizagens de indivíduos e grupos envolvidos, assumindo sua dimensão política e um fim social 29,38.

Metodologias participativas são utilizadas com o objetivo de ampliar a transparência das ações avaliadas e possibilitar aos envolvidos um repensar que possa facilitar mudanças 32 . Segundo a Organização Pan-Americana da Saúde (OPAS) ${ }^{39}$, a avaliação participativa é um processo contínuo de reflexão sobre o que foi realizado e obtido para orientar e modificar a ação futura. Essa reflexão se dá pela criação de ciclos contínuos de planejamento, monitoramento e avaliação. Mais que julgar o mérito de uma iniciativa de promoção da saúde, a proposta de uma metodologia participativa na condução de processos avaliativos é aprender com a iniciativa e com os atores envolvidos, documentar e analisar as mudanças ocorridas e aplicar as melhorias necessárias em longo prazo, favorecendo o empoderamento e a autonomia baseados na participação em um processo sustentável de desenvolvimento de capacidades, incorporando as lições aprendidas no processo de tomada de decisões.

Assim, a definição de aspectos metodológicos depende: do objetivo da avaliação e sua utilidade; das características da estratégia a ser avaliada, o que inclui não apenas aspectos técnicos e materiais, mas também a influência dos atores que a desenvolvem; da condição e envolvimento do avaliador, considerando as possibilidades de uma abordagem de avaliação participativa (avaliação interna), ou avaliação não participativa (avaliação externa); bem como do tipo de ações necessárias para alcançar os objetivos da avaliação, definidos pela delimitação da pergunta de avaliação, estratégias para coleta das informações e uso da informação/conhecimento produzido 35 .

Desenvolver capacidades em processos avaliativos é reiterar a centralidade da participação como conceito de promoção da saúde, disseminado por meio da Carta de Ottawa como: "um processo de capacitação das pessoas para aumentar o controle sobre sua saúde e melhorá-la" 40. Desenvolver capacidades decorre de um processo de médio e longo prazos que deve ser reinventado localmente e de acordo com o contexto do entorno 29,41. Requer o estabelecimento de estruturas de incentivos positivos e consistentes, pois significa crescer por intermédio das capacidades existentes, mais do que produzir novas. Desse modo, envolver profissionais da equipe de saúde bucal no processo de avaliação de estratégias de promoção da saúde bucal desenvolvidas em seus 
territórios de atuação caracteriza-se como uma estratégia metodológica importante para a qualificação das práticas.

\section{Explorando pilares e valores da promoção da saúde}

A literatura sobre promoção da saúde e avaliação de estratégias de promoção da saúde explora princípios e valores referenciais para a construção, desenvolvimento, avaliação de práticas promotoras da saúde além dos dilemas implicados com a avaliação de tais princípios e valores. A sistematização dessa literatura permitiu a construção de um modelo teórico, apresentado na Figura 1, que contempla os pilares e valores da promoção da saúde, o qual pode servir de base para a definição de princípios e estratégias avaliativas de práticas de promoção da saúde em diferentes contextos, incluindo aquelas produzidas nos territórios da atenção primária em saúde. A definição conceitual de pilares e valores foi sugerida por Takeda \& Harzheim, em 200642.

Neste modelo, os pilares entendidos como as bases teóricas para a indicação de valores da avaliação incluem a equidade, a participação e a sustentabilidade. Tais pilares seriam o esteio para os valores definidos como a âncora moral para as estratégias de promoção da saúde, que incluem a autonomia, o empoderamento, a integralidade, a intersetorialidade e a governança.

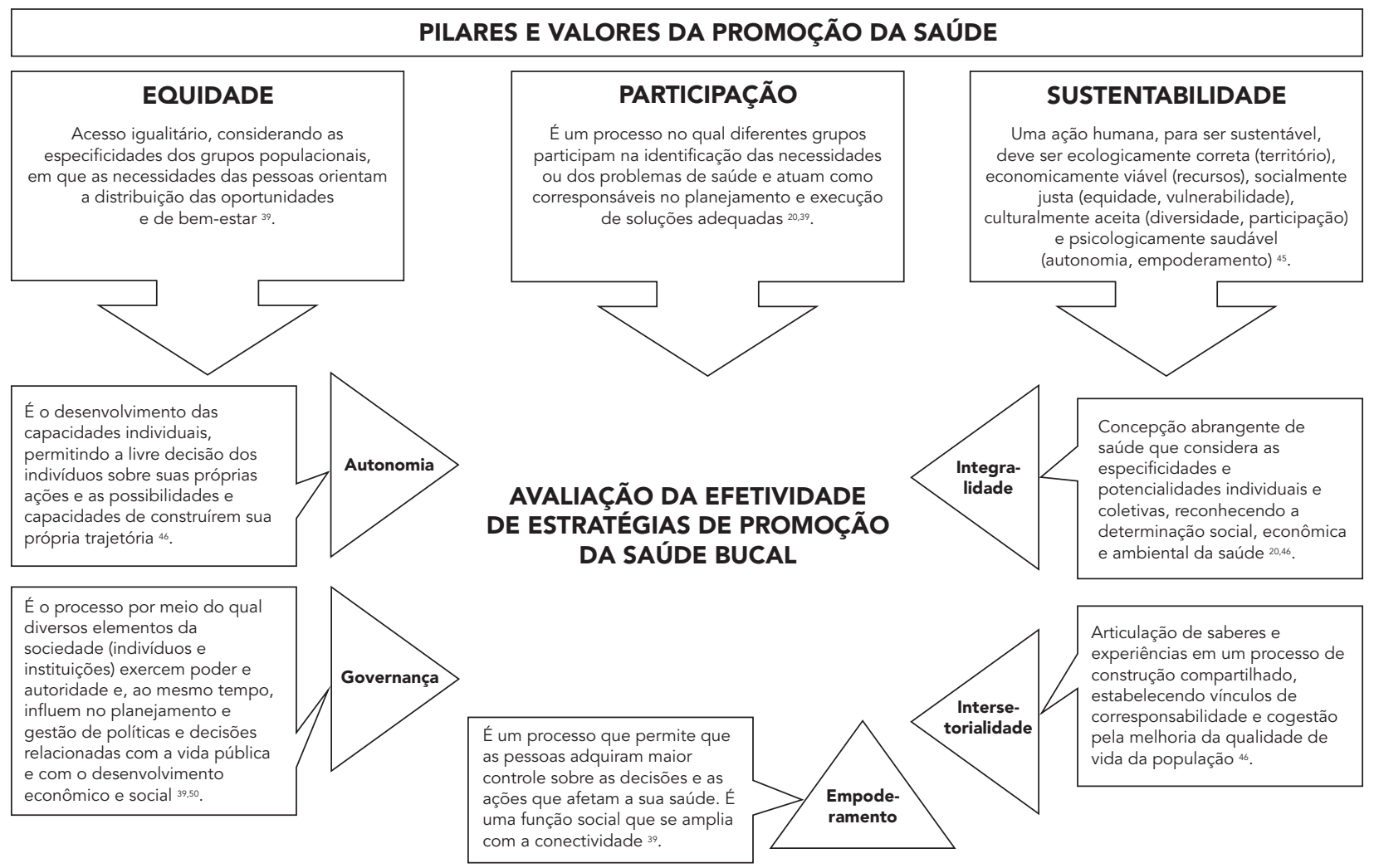

Fonte: trabalho de pesquisa. 
Aspectos conceituais relativos a esses pilares e valores são apresentados a seguir:

\section{Equidade}

A equidade, sendo princípio ético e conceito seminal, está na base de práticas de promoção da saúde. Alcançar equidade se constitui em um requisito fundamental para a saúde e para a qualidade de vida. Por isso o grande desafio da promoção da saúde bucal é a mudança de cenário, no qual ainda prevalecem as iniquidades sociais e de saúde, com deterioração das condições de vida da maioria da população, com o aumento de riscos para a saúde e diminuição dos recursos para enfrentá-los 43 .

A equidade centra-se na ideia de que todos devem ter justa oportunidade de obter seu pleno potencial e ninguém deveria ficar em desvantagem para alcançá-lo, se isso puder ser evitado. $\mathrm{O}$ reconhecimento das diferenças injustas que existem na sociedade em relação à saúde e a ação responsável sobre elas é ponto fundamental para a compreensão do conceito da equidade na perspectiva da promoção da saúde 44 .

\section{Participação}

É um processo em que diferentes grupos participam na identificação das necessidades ou dos problemas de saúde e atuam como corresponsáveis no planejamento e execução de soluções adequadas 20,39.

A participação envolve dimensões de negociação, informação, avaliação e monitoramento voltados ao desenvolvimento de estratégias resolutivas de atenção em saúde. A promoção da saúde pressupõe a organização coletiva e a busca de interesses comuns, envolvendo diferentes atores sociais, entidades e associações, sujeitos e lideranças parlamentares e outras representações sociais na construção de ações integradas 44 .

\section{Sustentabilidade}

Uma ação humana, para ser sustentável, deve ser ecologicamente correta, pensada na relação do homem com seu território e/ou ambiente; economicamente viável, com adequada utilização dos recursos disponíveis; socialmente justa, quando prioriza a vulnerabilidade de pessoas e grupos e busca equidade; culturalmente aceita, quando valoriza a diversidade e a participação; e psicologicamente saudável, favorecendo a autonomia e o empoderamento 45.

Na promoção da saúde, a sustentabilidade deve estar referida a um complexo sistema de relações que envolve atores e instituições na busca de soluções mais amplas e duradouras, e observar com mais atenção o conceito em relação aos arranjos societários que vão se estabelecendo e que podem permitir a longevidade das iniciativas. Aponta a necessidade de buscar caminhos articulados e de construir novas institucionalidades que ganhem potência e qualidade para enfrentar os desafios do cenário atual da atenção em saúde 44 .

\section{Autonomia}

A conquista progressiva da autonomia, tida como um componente desejável da promoção da saúde, é o desenvolvimento pessoal das capacidades, permitindo a livre decisão dos sujeitos sobre suas próprias ações e as possibilidades de construírem sua própria trajetória 46 . É fundamental reconhecer cada sujeito como um cidadão portador de direitos que deve ser respeitado em sua singularidade e ouvido quanto às suas necessidades em saúde 44 . Autonomia relaciona-se à ideia de liberdade, protagonismo, proatividade social, redes de proteção e cuidado recíproco, respeito à subjetividade e pluralidade de visões de mundo e comportamentos no âmbito local 47.

\section{Empoderamento}

O empoderamento (tradução de empowerment), às vezes também denominado potencialização, é um processo que permite que as pessoas adquiram maior controle sobre as decisões e as ações que afetam a sua saúde 39 .

A noção de empoderamento remete ao fortalecimento independente das populações, portanto não pode ser concedido ou estimulado por relações assimétricas de poder. É uma estratégia importante de promoção da saúde que pode ajudar a transformar a realidade por meio do fortalecimento econômico, político, social e cultural dos múltiplos atores sociais que, em conjunto, possam compreender a determinação do processo saúde-doença e conquistar o direito à vida com ética e dignidade humana 28,43 .

É, pois, um processo dinâmico do desenvolvimento político e tomada de consciência crítica de uma população, envolvendo o respeito recíproco, a participação solidária e o cuidado do grupo, por meio dos quais se compartilham saberes e poderes $29,48,49$.

\section{Governança}

É o processo pelo qual diversos elementos da sociedade (indivíduos e instituições) exercem poder e autoridade e, ao mesmo tempo, influenciam no planejamento e gestão de políticas pú- 
blicas, como estratégias de promoção da saúde, afetando decisões relacionadas à vida pública e ao desenvolvimento social e humano 39,50.

\section{Integralidade}

Concepção abrangente de saúde que considera as especificidades e potencialidades individuais e coletivas, reconhecendo a determinação social, econômica e ambiental da saúde 20,46.

A integralidade deve ser assumida como um eixo norteador de novas formas de produção social da saúde, sendo paralelamente uma nova forma de gestão de cuidados nas instituições de saúde, permitindo o surgimento de experiências inovadoras na incorporação e desenvolvimento de novas tecnologias assistenciais 51,52 .

\section{Intersetorialidade}

Ações intersetoriais voltadas para a promoção da saúde implicam a articulação de saberes e experiências em um processo de construção compartilhada, de troca e construção coletiva com base em diferentes lugares, culturas institucionais e linguagens. Impõe o equacionamento muitas vezes conflitivo de relações de poder e de distintas perspectivas de práticas entre diversos sujeitos e setores envolvidos na tentativa de enfrentar questões sanitárias, mas cuja superação e transcendência permitem soluções inovadoras e duradouras, tanto quanto o estabelecimento de vínculos de corresponsabilidade e cogestão para a melhoria da qualidade de vida de forma democrática e resolutiva 3,46 .

\section{Operacionalizando conceitos}

Como traduzir tais conceitos para nortear processos avaliativos de estratégias de promoção da saúde bucal desenvolvidas nos contextos da atenção primária em saúde no Brasil?

A prática reflexiva inicial exige que se construam, mediante os pilares e valores da promoção da saúde, referenciais para que sejam exploradas as complexas características de práticas com potencial para atuar sobre determinantes ampliados da saúde bucal e provocar mudanças sustentáveis no perfil da condição de saúde das populações cobertas. Talvez um bom ponto de partida seja a identificação do que não é promoção da saúde: concepções e práticas que caminham para lados opostos aos reconhecidos pilares e valores da promoção da saúde descritos anteriormente. Por exemplo, práticas que não busquem a garantia do direito à saúde com equidade, que não contemplem oportunidades de participação real daqueles mais diretamente interessados em seus resultados, bem como práticas pontuais, desconectadas e descompromissadas da realidade local sendo, portanto, não sustentáveis.

Sistematizar os conhecimentos, valendose do modelo teórico proposto, em uma ferramenta de avaliação da efetividade de práticas de promoção da saúde bucal, considerando sua adequação aos pilares e valores identificados, traduz-se em um esforço para operacionalizar conceitos. Pode ser um importante suporte para o desenvolvimento de capacidades locais e para a instrumentalização de profissionais e gestores da saúde para o reconhecimento de ações com potencialidade para promover saúde bucal e para a tomada de decisão informada nos diferentes territórios de atuação.

Assim, por esse processo de sistematização, apresenta-se a seguir uma matriz inicial de descritores, definidos por Deslandes \& Lemos 53 como o conteúdo, os pontos críticos e as características significativas consideradas como foco central para a avaliação. Tais descritores foram organizados em três grandes dimensões relacionadas aos principais ganhos esperados: a saúde bucal, a construção de políticas públicas saudáveis e o desenvolvimento humano e social.

A saúde bucal foi aqui entendida como um resultado de ações que compreendem a saúde como um recurso para a vida com qualidade, determinada pelas condições sociais e ambientais de vida na comunidade, e construída mediante a relação positiva da boca humana com o corpo biológico de homens e mulheres e deles com o corpo social 54. Políticas públicas saudáveis compreendem ações que favoreçam, por meio de estratégias populacionais, a criação de um ambiente social e físico de apoio, potencializador da saúde, possibilitando e facilitando que as pessoas façam escolhas saudáveis 39 . Como ganho ampliado, o desenvolvimento humano e social foi aqui considerado como ações favorecedoras para que a sociedade seja composta cada vez mais por sujeitos felizes, saudáveis, criativos, produtivos e que promovam o cuidado e a paz, com saúde bucal 55 .

A Tabela 1 apresenta a matriz de descritores e dimensões sistematizadas para avaliação de estratégias de promoção da saúde bucal desenvolvidas nos territórios da atenção primária à saúde.

Essa matriz pode nortear a construção de indicadores que permitam mensurar e qualificar a adequação das práticas avaliadas aos pilares e valores da promoção da saúde, além de apoiar técnicos e gestores na formulação de novas práticas balizadas e com potencial para provocar 
Tabela 1

Matriz de descritores.

\begin{tabular}{|c|c|c|}
\hline Saúde bucal & Políticas públicas saudáveis & Desenvolvimento humano e social \\
\hline $\begin{array}{l}\text { 1. Reconhecimento dos Determinantes Sociais da } \\
\text { Saúde no desenvolvimento da estratégia avaliada }\end{array}$ & $\begin{array}{l}\text { 1. Priorização de grupos mais vulneráveis } \\
\text { 2. Participação equânime da população alvo }\end{array}$ & $\begin{array}{l}\text { 1. Reconhecimento de valores da população alvo } \\
\text { para o desenvolvimento da estratégia }\end{array}$ \\
\hline $\begin{array}{l}\text { 2. Reconhecimento de fatores comuns de risco } \\
\text { 3. Acesso ao perfil epidemiológico de doenças } \\
\text { e agravos bucais antes e durante o desenvolvi- } \\
\text { mento da estratégia }\end{array}$ & $\begin{array}{l}\text { 4. Parceria entre diferentes profissionais de saúde } \\
\text { no território }\end{array}$ & $\begin{array}{l}\text { 2. Participação da comunidade na definição de } \\
\text { prioridades, objetivos, condução e avaliação da } \\
\text { estratégia }\end{array}$ \\
\hline $\begin{array}{l}\text { 4. Comparação e reconhecimento de resultados } \\
\text { alcançados }\end{array}$ & $\begin{array}{l}\text { 5. Parceria com outras atividades de promoção } \\
\text { da saúde no território }\end{array}$ & $\begin{array}{l}\text { 4. Mudanças no processo de condução da estra- } \\
\text { tégia com base nas sugestões da comunidade }\end{array}$ \\
\hline \multirow[t]{5}{*}{$\begin{array}{l}\text { 5. Potencialização da definição de novos objetivos } \\
\text { da estratégia }\end{array}$} & $\begin{array}{l}\text { 6. Reconhecimento e apoio da estratégia pela } \\
\text { gerência de saúde local, distrital e municipal }\end{array}$ & $\begin{array}{l}\text { 5. Divulgação da estratégia para a comunidade } \\
\text { local }\end{array}$ \\
\hline & $\begin{array}{l}\text { 7. Recursos específicos alocados para o desen- } \\
\text { volvimento da estratégia }\end{array}$ & 6. Protagonismo compartilhado da estratégia \\
\hline & $\begin{array}{l}\text { 8. Previsão de avaliações de processo e resulta- } \\
\text { dos ao longo do desenvolvimento da estratégia }\end{array}$ & $\begin{array}{l}\text { 7. Desenvolvimento de ações de educação } \\
\text { permanentes para profissionais de saúde e difer- } \\
\text { entes atores sociais }\end{array}$ \\
\hline & $\begin{array}{l}\text { 9. Pactuação e reconhecimento dos resultados } \\
\text { pela Unidade de Saúde local }\end{array}$ & $\begin{array}{l}\text { 8. Manutenção dos resultados e benefícios da } \\
\text { estratégia }\end{array}$ \\
\hline & $\begin{array}{l}\text { 10. Pactuação e reconhecimento dos resultados } \\
\text { pelo Conselho Local de Saúde }\end{array}$ & \\
\hline
\end{tabular}

mudanças no perfil de saúde bucal da população brasileira.

\section{Considerações finais}

Avaliar estratégias de promoção da saúde bucal é um desafio apontado por gestores, técnicos e pesquisadores envolvidos com a atenção primária à saúde. A avaliação dessas práticas pressupõe uma abordagem metodológica participativa e definida com base no reconhecimento de fatores contextuais complexos relacionados ao perfil social, político, cultural e organizacional em que as práticas se desenvolvem.

Potencializar estratégias de promoção da saúde bucal para alcançar melhores resultados é parte das diretrizes da atual Política Nacional de Saúde Bucal e Vigilância em Saúde Bucal. A construção de referenciais consistentes para avaliação de tais estratégias, baseados nos pilares e valores da promoção da saúde, pode apoiar processos avaliativos que permitam o reconhecimento, fortalecimento e mudanças nas estratégias com potencial para promover a saúde bucal.

É importante a validação de instrumentos que possam se tornar ferramentas úteis e relevantes à construção de capacidades locais e instrumentalizar profissionais e gestores da saúde para tomada de decisão nos diferentes territórios de atuação. Dessa forma, escolhas político-teóricas coerentes e baseadas nas melhores evidências disponíveis sobre a efetividade das intervenções planejadas podem ser contextualizadas na atenção primária em saúde. 


\section{Resumo}

A avaliação de ações de promoção da saúde é um desafio metodológico e estratégico para a construção de evidências que possam apoiar processos de gestão em saúde. O emprego de métodos adequados de avaliação, baseado na análise participativa de processos e contextos locais, é indispensável ao sucesso das intervençães e formulação e implementação de políticas. A Política de Promoção e Vigilância em Saúde Bucal no Brasil explicita a necessidade de aprimorar estratégias avaliativas de ações de promoção da saúde bucal desenvolvidas no contexto da atenção primária que permitam avaliar não somente seus resultados e impacto, mas também o processo político e social para alcançar os objetivos estabelecidos. Este artigo se propõe a sistematizar a literatura no campo da avaliação da efetividade de estratégias de promoção da saúde, definir um modelo teórico e propor uma matriz de descritores, explorando a base referencial da promoção da saúde e práticas com potencialidade para reduzir situações de fragilidade de grupos populacionais, combater iniquidades e incorporar a participação na gestão da saúde.

Promoção da Saúde; Atenção Primária à Saúde; Saúde Bucal

\section{Referências}

1. Moysés SJ. O futuro da Odontologia, no Brasil e no mundo, sob o ponto de vista da Promoção da Saúde (Opinião). Rev Bras Odontol 2008; 16:10-3.

2. Agência Nacional de Saúde Suplementar. Manual técnico - promoção da saúde e prevenção de riscos e doenças na saúde suplementar. 2a Ed. Brasília: Agência Nacional de Saúde Suplementar/ Ministério da Saúde; 2007.

3. Moretti AC, Teixeira FF, Suss FMB, Lima LSM, Bueno RE, Moysés SJ, et al. Intersetorialidade nas ações de promoção de saúde realizadas pelas equipes de saúde bucal de Curitiba (PR). Ciênc Saúde Coletiva 2010; 15 Suppl 1:1827-34.

4. Santos APP, Nadanovski P, Oliveira BH. Survey of Brazilian governmental health agencies shows conflicting recommendations concerning oral hygiene practices for children. Cad Saúde Pública 2010; 26:1457-63.

\section{Colaboradores}

S. Z. Kusma, S. T. Moysés e S. J. Moysés contribuíram igualmente na construção do artigo.

\section{Agradecimentos}

Agradecemos à Coordenação Nacional de Saúde Bucal do Ministério da Saúde e Fundo Nacional de Saúde, ao Prof. Dr. Paulo Sávio A. de Goes (Universidade de Pernambuco) por sua contribuição no desenvolvimento deste trabalho, aos participantes da Oficina de Validação do Construto e a todos os profissionais cirurgiõesdentistas atuantes no Sistema Único de Saúde da Prefeitura Municipal de Curitiba.
5. Brown L. Research in dental health education and health promotion: a review of the literature. Health Educ Q 1994; 21:83-102.

6. Schou L, Locker D. Oral health: a review of the effectiveness of health education and health promotion. Amsterdam: Dutch Centre for Health Promotion and Health Education; 1994.

7. Kay L, Locker D. Is dental health education effective? A sistematic review of current evidence. Community Dent Oral Epidemiol 1996; 24:231-5.

8. Sprod AJ, Anderson R, Treasure ET. Effective oral health promotion: literature review. Cardiff: Dental Public Health Unit, University of Wales College of Medicine; 1996. (Health Promotion Wales Technical Report, 20).

9. Kay L, Locker D. A sistematic review of the effectiveness of health promotion aimed at promoting oral health. London: Health Education Authority; 1997. 
10. Watt RG. Strategies and approaches in oral disease prevention and health promotion. Bull World Health Organ 2005; 83:711-8.

11. Watt RG. From victim blaming to upstream action: tackling the social determinants of oral health inequalities. Community Dent Oral Epidemiol 2007; 35:1-11.

12. Secretaria de Vigilância em Saúde, Ministério da Saúde. Vigilância em saúde no SUS - fortalecendo a capacidade de resposta aos velhos e aos novos desafios. Brasília: Ministério da Saúde; 2006.

13. Watt RG, Harnett R, Daly B, Fuller SS, Kay E, Morgan A, et al. Evaluating oral health promotion: need for quality outcomes measures. Community Dent Oral Epidemiol 2006; 34:11-7.

14. Scriven A, Speller V. Global issues and challenges beyond Ottawa: the way forward. Promot Educ 2007; 14:194-8.

15. Coordenação Nacional de Saúde Bucal, Secretaria de Atenção à Saúde, Ministério da Saúde. Diretrizes da Política Nacional de Saúde Bucal. Brasília: Ministério da Saúde; 2004.

16. Westphal MF, Mendes R. Cidade saudável: uma experiência de interdisciplinaridade e intersetorialidade. Rev Adm Pública 2000; 34:47-62.

17. Naidoo J, Wills J. Health promotion: foundations for practice. 2nd Ed. London: Baillière Tindall; 2003.

18. Pedrosa JIS. Perspectivas na avaliação em promoção da saúde: uma abordagem institucional. Ciênc Saúde Coletiva 2004; 9:617-26.

19. Petersen PE. Priorities for research for oral health in the 21st century: the approach of the WHO Global Oral Health Programme. Community Dent Health 2005; 22:71-4.

20. Ministério da Saúde. Política Nacional de Promoção da Saúde. Brasília: Ministério da Saúde; 2006.

21. De Salazar L. Evaluación de efectividad en promoción de la salud. Guia de evaluación rápida. Santiago de Cali: Centro para el Desarrollo y Evaluación de Políticas y Tecnología en Salud Pública; 2004.

22. McQueen DV, Jones CM. Global perspectives on health promotion effectiveness. New York: Springer; 2007.

23. Ayres JR. Norma e formação: horizontes filosóficos para as práticas de avaliação no contexto da promoção da saúde. Ciênc Saúde Coletiva 2004; 9:583-92.

24. Brandão D, Silva RR, Palos CM. Da construção de capacidade avaliatória em iniciativas sociais: algumas reflexões. Ensaio: Avaliação e Políticas Públicas em Educação 2005; 13:361-73.

25. Hartz ZMA, Silva LMV. Avaliação em saúde: dos métodos teóricos à prática na avaliação de programas e sistemas de saúde. Salvador: EDUFBA/Rio de Janeiro: Editora Fiocruz; 2005.

26. Kleba ME, Wendhausen ALP. O processo de pesquisa como espaço e processo de empoderamento. Interface Comum Saúde Educ 2010; 14:427-36.

27. Moysés SJ, Moysés ST, Krempel MC. Avaliando o processo de construção de políticas públicas de promoção de saúde: a experiência de Curitiba. Ciênc Saúde Coletiva 2004; 9:627-41.
28. Westphal MF, Wallerstein N. Saúde, desenvolvimento e equidade. Promot Educ 2007; Supplement $1: 31-2$.

29. Franco de Sá R, Moysés ST. O processo avaliativo em promoção de saúde como estratégia de empoderamento e de desenvolvimento de capacidades. Boletim Técnico do Senac 2009; 35:29-35.

30. Nutbean D. Eficácia de la promoción de la salud: las perguntas que debemos responder. In: Unión Internacional de Promoción de la Salud y Educación para la Salud, editor. La evidencia de la eficacia de la promoción de la salud. Madrid: Ministério de la Salud y Consumo; 1999. p. 1-11.

31. De Salazar L. Evaluación de efectividad en promoción de la salud y salud pública. In: De Salazar L, editor. Efectividad en promoción de la salud y salud pública. Cali: Programa Editorial Universidad del Valle; 2009. p. 60-81.

32. Westphal MF, Mendes R. Avaliação participativa e a efetividade da promoção da saúde: desafios e oportunidades. Boletim Técnico do Senac 2009; 35:17-27.

33. Potvin L, Chabot P. Splendor and misery of epidemiology for the evaluation of health promotion. Rev Bras Epidemiol 2002; 5 Suppl 1:91-102.

34. Souza EM. Evaluation methods in health promotion programmes: the description of a triangulation in Brazil. Ciênc Saúde Coletiva 2010; 15 : 2521-32.

35. Potvin L, Bisset S. There is more to methodology than method. In: Potvin L, McQueen DV, editors. Health promotion evaluation practices in the America: values and research. New York: Springer; 2008. p. 63-77.

36. Unión Internacional de Promoción de la Salud e Educación para la Salud para la Comisión Europea. La evidencia de la efectividad de la promoción de la salud. Configurando la salud pública en una nueva Europa. Madrid: Rumagraf; 2000.

37. Centro para el Desarrollo y Evaluación de Políticas y Tecnología en Salud Pública. Guia para sistematización de intervenciones en promoción de la salud y salud pública. Cali: Universidade del Valle; 2008.

38. De Salazar L. Feasibility for health promotion under various decision-making contexts. In: McQueen DV, Jones CM, editors. Global perspectives on health promotion effectiveness. New York: Springer; 2007. p. 353-65.

39. Organización Panamericana de la Salud. Guia de evaluación participativa para municipios y comunidades saludables. Lima: Organización Panamericana de la Salud; 2005.

40. Organização Mundial da Saúde. Carta de Ottawa Primeira Conferência Internacional de Promoção da Saúde. Ottawa: Organização Mundial da Saúde; 1986.

41. Gaetani F. Desafios à gestão na cooperação internacional. Brasília: Programa das Nações Unidas para o Desenvolvimento; 2005. 
42. Takeda S, Harzheim E. Valores, princípios e atributos de um sistema de saúde orientado pela APS, OPAS, 2006. Problematizando os atributos fundamentais da APS/ESF - Grupo de Pesquisa em Atenção Primária à Saúde. Porto Alegre: Universidade Federal do Rio Grande do Sul; 2008.

43. Heidmann ITSB, Almeida MCP, Boehs AE, Wosny AM, Monticelli M. Promoção à saúde: trajetória histórica de suas concepções. Texto Contexto Enferm 2006; 15:352-8.

44. Castro AM, Sperandio AMG, Gosh CS, Rocha DG, Cruz DKA, Malta DC, et al. Curso de extensão para gestores do SUS em promoção da saúde. Brasília: Centro de Ensino a Distância/Fundação Universidade de Brasília; 2010.

45. United Nations. Report of the World Submit on Susteinable Development. Johannesburg: United Nations; 2002.

46. Westphal MF. Promoção da saúde e prevenção de doenças. In: Minayo MCS, Campos GWS, Akerman M, Drumond Júnior M, Carvalho YM, organizadores. Tratado de saúde coletiva. São Paulo: Editora Hucitec/Rio de Janeiro: Editora Fiocruz; 2006. p. 635-67.

47. Santos L, Da Ros M, Crepaldi M. Grupos de promoção à saúde no desenvolvimento da autonomia, condições de vida e saúde. Rev Saúde Pública 2006; 40:346-52.

48. Carvalho SR. Os múltiplos sentidos da categoria "empowerment" no projeto de Promoção à Saúde. Cad Saúde Pública 2004; 20:1088-95.
49. Oakley P, Clayton A. Monitoramento e avaliação de empoderamento. São Paulo: Instituto Pólis; 2003.

50. United Nations Human Settlements Programme. The global campaign on urban governance: concept paper. Nairobi: United Nations Human Settlements Programme; 2002.

51. Hartz ZMA. Atenção básica e integralidade: contribuições para estudos de práticas avaliativas em saúde. Ciênc Saúde Coletiva 2009; 14 Suppl 1:1625-7.

52. Pinheiro R, Silva Jr. A, Mattos R. Atenção básica e integralidade: contribuições para estudos de práticas avaliativas em saúde. Rio de Janeiro: Instituto de Medicina Social, Universidade do Estado do Rio de Janeiro/ABRASCO; 2008.

53. Deslandes SF, Lemos MP. Construção participativa de descritores para avaliação dos núcleos de prevenção de acidentes e violência, Brasil. Rev Panam Salud Pública 2008; 24:441-8.

54. Moysés SJ. Saúde bucal. In: Giovanella L, Escorel S, Lobato L, editores. Políticas e sistema de saúde no Brasil. Rio de Janeiro: Editora Fiocruz; 2008. p. 705-34.

55. Hancock T, editor. Conceptual model healthy community. II Oficina de Ações Intersetoriais de Promoção da Saúde. Recife: Projeto Ações Intersetoriais para a Saúde; 2009.

Recebido em 28/Dez/2010

Versão final reapresentada em 26/Jun/2011

Aprovado em 06/Jul/2011 